\title{
SPATIO-TEMPORAL TOPOLOGICAL RELATIONSHIPS BETWEEN LAND PARCELS IN CADASTRAL DATABASE
}

\author{
Wei Song ${ }^{\text {a, } *, \text { Fu Zhang }}{ }^{\text {a }}$ \\ ${ }^{a}$ School of Resources and Environment, North China University of Water Resources and Electric Power, \\ Zhengzhou, China -zzsongwei@ hotmail.com
}

\section{Commission VI}

\begin{abstract}
KEY WORDS: Spatio-temporal topological relationships, Spatial topological relationships, Temporal topological relationships,
\end{abstract} Land parcel

\begin{abstract}
:
There are complex spatio-temporal relationships among cadastral entities. Cadastral spatio-temporal data model should not only describe the data structure of cadastral objects, but also express cadastral spatio-temporal relationships between cadastral objects. In the past, many experts and scholars have proposed a variety of cadastral spatio-temporal data models, but few of them concentrated on the representation of spatiotemporal relationships and few of them make systematic studies on spatiotemporal relationships between cadastral objects. The studies on spatio-temporal topological relationships are not abundant. In the paper, we initially review current approaches to the studies of spatio-temporal topological relationships, and argue that spatio-temporal topological relation is the combination of temporal topology on the time dimension and spatial topology on the spatial dimension. Subsequently, we discuss and develop an integrated representation of spatio-temporal topological relationships within a 3-dimensional temporal space. In the end, based on the semantics of spatiotemporal changes between land parcels, we conclude the possible spatio-temporal topological relations between land parcels, which provide the theoretical basis for creating, updating and maintaining of land parcels in the cadastral database.
\end{abstract}

\section{INTRODUCTION}

Cadastral spatial entities, such as land parcel, boundary line, boundary point, etc, have distinct spatial and temporal characteristics. Land parcel is the most important entity in the cadastral domain, which not only occupy a certain position in space, and change frequently with the change of time. Numerous experts and scholars have made many efforts to study on Cadastral spatio-temporal data management, and proposed many cadastral spatio-temporal data model (Fan, et al., 2009). However, few of them made a systematic study on the spatio-temporal topological relationships between land parcels. CHANG, et al. (1997) discovered the properties of spatiooverlap and time-meet between the successive land parcels by analyzing the spatio-temporal process of land subdivision. ZHOU, et al. (2003) proposed one extended 4-intersection method of spatial topological relations between a simple land parcel and a land parcel with one hole, which used double 4intersection arrays to describe the spatial topological relations. FENG, et al. (2006) proposed the topological relationships test method based on the spatial topological rules of cadastral objects. XU, et al. (2002) discussed the cadastral change types and analyzed the spatial topological relationships between land parcels. WU, et al. (2012) analyzed the characteristic of land parcel change and concluded six conditions which temporal land parcels should meet.

Although many efforts have been made by numerous researchers to describe and express the spatio-temporal topological relationships between land parcels, there are some shortcomings. One is that most of researchers are only focus on the spatial topological relationships between land parcels, such as ZHOU, et al. (2003), FENG, et al. (2006). The other is that the study of spatio-temporal topological relationships is not systematic and incomplete, such as CHANG, et al. (1997), XU, et al. (2002), WU, et al. (2012).

In the paper, we argue that spatio-temporal topological relationship is the combination of temporal topology on the time dimension and spatial topology on the spatial dimension. Subsequently, we develop an integrated representation of spatio-temporal topological relationships within a 3dimensional temporal space. In the end, based on the semantics of spatiotemporal changes of land parcels, we conclude the possible spatio-temporal topological relations between land parcels. And we summary the possible spatio-temporal topological relationships among current land parcels, history land parcels, and both of them, which provide the theoretical basis for creating, updating and maintaining of land parcels in the cadastral database.

The remainder of this paper is structured as follows. Cadastral objects and their change types in cadastral database are analyzed in Section 2. Representation of spatio-temporal topological relationships between land parcels is presented in Section 3. The summary is given in Section 4.

\section{LAND PARCEL AND ITS CHANGE TYPES}

\subsection{Land parcel in cadastral management}

Land parcel is the smallest saleable area, and the geometric feature is equivalent to polygon in cadastral systems. Land parcels are composed of boundary points and boundary lines. Although many entities in cadastral management, land parcel is the basic unit and the most important entity in cadastral management.

\footnotetext{
* Corresponding author.
} 
According to the object-oriented view, land parcel is described as $\mathrm{P}\{\mathrm{OID}, \operatorname{Attr}(\mathrm{t}), \mathrm{S}, \mathrm{T}$, Actions $\}$, where OID is the unique identifier of a land parcel, $\operatorname{Attr}(t)$ is the non-spatial attributes of a land parcel, $\mathrm{S}$ is the spatial attribute of a land parcel, $\mathrm{T}$ shows its life span. Suppose P is a land parcel in the cadastral space, which is created on ts, then change on te because of the event of land change, thus the life span of land parcel $\mathrm{P}$ is expressed as $\mathrm{L}=[\mathrm{ts}, \mathrm{te}]$.

\subsection{Change of land parcels}

Land parcels are of dynamic nature, as they change in space and time for a number of reasons. Therefore, land parcels can be described as spatio-temporal object, which change frequently with time. Land parcel is given birth through land initial registration and is changed through the change registration process. Land parcel's change is categorized into three types according to its change contents: (1) Spatial change, (2) Thematic attributes change, and (3) Spatial and thematic attribute change.

Generally, there are four fundamental cases which result in the change of land parcel's geometry or topology (Fig.1). They can be described as follows: (a) split (Fig.1.a); (b) union, (Fig.1.b); (c) reallocation (Fig.1.c); (d) modification of the common border (Fig.1.d).

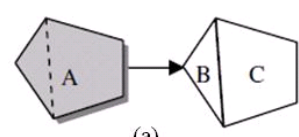

(a)

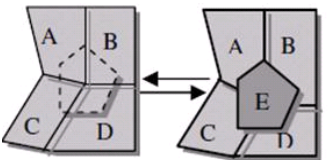

(c)

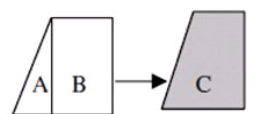

(b)

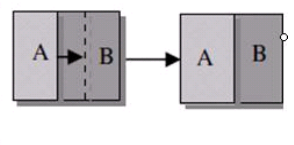

(d)
Figure 1.Spatial change of land parcel.

\subsection{Current Land Parcels Set and Historic Land Parcels Set}

According to the view of Temporal Geographic Information System, each geographic entity has a starting and ending instant. The time interval which a geographic entity exist in the real world is named as Life span. Similarly, a land parcel also has a starting and ending date in time. When the spatial attribute of one or more land parcel change in the cadastral management, the land parcel(s) will disappear, and one or more land parcel will be produced. The previous land parcel(s) are usually called as historic land parcel. And the new land parcel(s) are called as current land parcel. We usually store current land parcel in the current land parcel set, and store historic land parcel in the historic land parcel set.

Definition 1.Current Land Parcel Set: Suppose Pi is a land parcel in the cadastral space, which is created on t $j$ and still exists at the time instant $\mathrm{c}$, where $\mathrm{c}$ denotes the current time. We name land parcel Pi as current land parcel. All of land parcels which exist at this time instant are stored in Current Land Parcel Set, which is denoted as $\mathrm{CP}=\{\mathrm{CP} 1, \mathrm{CP} 2, \ldots \mathrm{CPi}, \ldots, \mathrm{CPn}\}$, where $\mathrm{CPi}$ denote any land parcel which exists at the current time.

Definition 2.Historic Land Parcel Set: Suppose Pi is a land parcel in the cadastral space, which is created on time instant $t j$ and disappear on time instant tk (tk is after $\mathrm{tj}$ ). We name land parcel Pi as historic land parcel. All of historic land parcels are stored in the Historic Land Parcel Set, which is denoted as HP=
$\{\mathrm{HP} 1, \mathrm{HP} 2, \ldots \mathrm{HPi}, \ldots, \mathrm{HPn}\}$, where HPi denote any historic land parcel.

Current Land Parcel Set includes land parcels which have specific starting time and still exist at this moment. Historic Land Parcel Set includes land parcels which have a specific life span and have become history files in cadastral management.

\section{REPRESENTATION OF SPATIO-TEMPORAL TOPOLOGICAL RELATIONSHIPS BETWEEN LAND PARCELS}

\subsection{Spatio-temporal topological relationships}

The description and judge of spatio-temporal topological relationships between spatial entities is important for spatiotemporal data modeling, spatio-temporal query and analysis, spatio-temporal reasoning, data updating and data consistency check in the spatial database. Spatio-temporal topological relationship is a special relation between spatial entities in the world. Over the past years, many researchers in different communities such as GIS, Artificial intelligence and Spatiotemporal Database make many efforts to study it. SHU, et al. (1997) presented and proved a 4I framework to describe temporal topological relationships completely and uniquely, and listed a set of predicates describing temporal topological relationships, distinguished the intention and the predicate description form of temporal topological relationships and time direction relationships . Claramunt, et al. (2001) introduced a new approach that combines topological relationships between regions in 2-dimensional space with temporal relationships between convex intervals in time. Resulting relationships are defined and visually presented within a 3-dimensional space that integrates the geographical space as a 2-dimensional space and the time line as the third dimension. XUE, et al. (2008) proposed an integrated spatio-temporal framework based on Cartesian operation, and concluded 64 kinds of jointly exhaustive and pairwise disjoint spatio-temporal topologies. Zeng, et al. (2010) defined a qualitative model for the spatiotemporal topological relations between temporal GIS objects by using 9-Intersection model and time expression based on timeslice, and discussed the computation rules for spatio-temporal topological relations. SHEN, et al. (2010) proposed the integration framework for spatial-temporal topological relationships on the analysis of Allen's 13 temporal topological relationships and Egenhofer's 8 spatial relationships on regions, and concluded 104 kinds of spatio-temporal topological relationships between spatio-temporal objects.

Spatio-temporal topological relationship is the combination of the spatial topological relationships in space with temporal topological relationships in time. Spatial topological relationship is one of the important issues in the researches on GIS and spatial databases. Until now, many people studied this issue and got some valuable results, which can be categorized into two groups (i.e. the formal model of RCC and the nintersection model based on point set theory). Egenhofer studied the spatial topological relations and defined 8 kinds of spatial topological relationships between two convex regions using 4intersection model based on the point set theory, i.e.\{Disjoint, Meet, Overlap, Cover, Coveredby, Equal, Inside, Contain $\}$ (Egenhofer, et al.,2001). In this paper, we record 8 kinds of spatial topological relationships as $\mathrm{RP}\left\{\mathrm{R}_{\text {Disjoint }}, \mathrm{R}_{\text {Meet }}, \mathrm{R}_{\text {Overlap}}\right.$, $\left.\mathrm{R}_{\text {Cover }}, \mathrm{R}_{\text {Coveredby, }}, \mathrm{R}_{\text {Equal }}, \mathrm{R}_{\text {Inside }}, \mathrm{R}_{\text {Contain }}\right\}$.

Temporal relations are isomorphic to the spatial relations. These relations are defined as using metric, topological, and ordertheory concepts. James Allen defined 13 binary relationships between convex temporal intervals (Allen, et al., 1984). SHU 
(1997) concluded 8 kinds of temporal topological relationships $\left\{\mathrm{T}_{\text {Disjoint }}, \mathrm{T}_{\text {Meet }}, \mathrm{T}_{\text {Overlap }}, \mathrm{T}_{\text {Cover }}, \mathrm{T}_{\text {Coveredby }}, \mathrm{T}_{\text {Equal }}, \mathrm{T}_{\text {Inside }}, \mathrm{T}_{\text {Contain }}\right\}$ based on the theory of the point set. In this paper, we record 8 kinds of temporal topological relationships as $\mathrm{TP}\left\{\mathrm{T}_{\text {Disjoint }}, \mathrm{T}_{\text {Meet }}\right.$, $\left.\mathrm{T}_{\text {Overlap }}, \mathrm{T}_{\text {Cover }}, \mathrm{T}_{\text {Coveredby }}, \mathrm{T}_{\text {Equal }}, \mathrm{T}_{\text {Inside }}, \mathrm{T}_{\text {Contain }}\right\}$.

\subsection{Representation of Spatio-temporal topological relationships between land parcels}

Land parcel is a polygon located in space, which is referenced by a convex interval of time. The spatial topological relationships between land parcels change frequently with time. The spatio-temporal topological relationship is the combination of temporal topology on the time dimension and spatial topology on the spatial dimension. Inspired by the representation of spatial and temporal relationships between evolving regions of Claramunt Christophe (2001) and XUE (2008), we construct a 3-dimensional cadastral space, which spatial topological relationships are represented in a horizontal plane, and the representation of temporal topological relationships are in the vertical axis. There are 8 kinds of spatial topological relationships between land parcels, and 8 kinds of temporal topological relationships between land parcels. Therefore, we can conclude that there are $8 \times 8$ spatio-temporal topological relationships between two land parcels. Figure 2 introduces a graphic presentation of the resulting 3-dimensional relationships that represent the cross product of these topological and temporal relationships between land parcels.

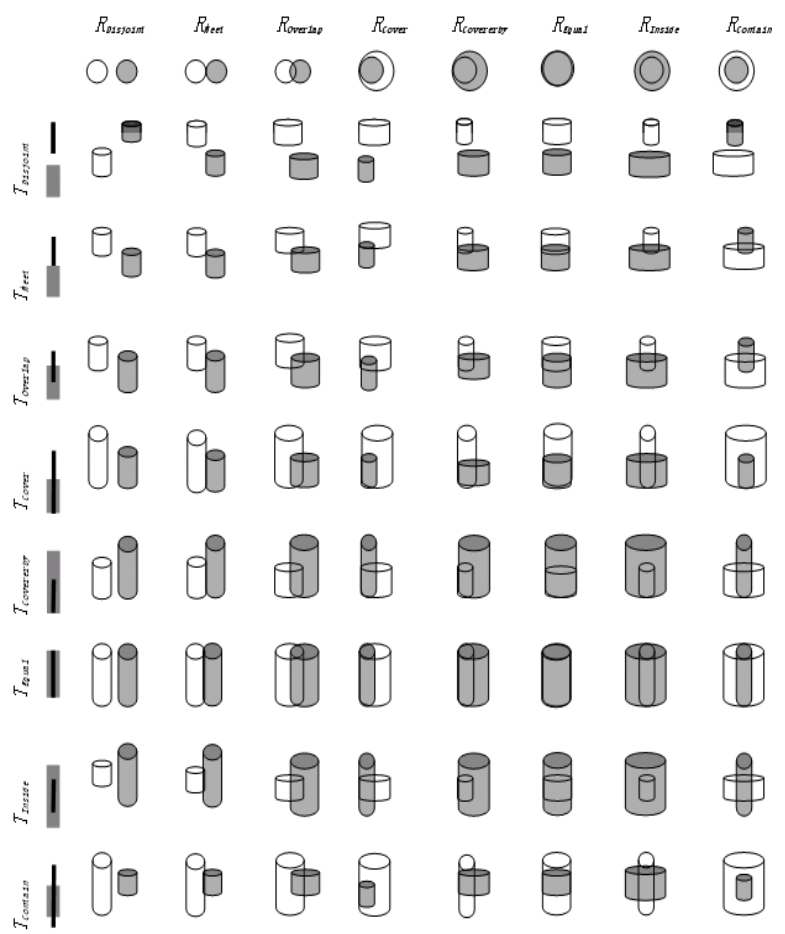

Figure 2. spatio-temporal relationships between land parcels in cadastral temporal space

From the above discussion, there are 64 spatio-temporal topological relationships between land parcels. We can describe them as $\left\{\mathrm{R}_{\text {Disjoint }} \mathrm{T}_{\text {Disjoint }}, \mathrm{R}_{\text {Disjoint }} \mathrm{T}_{\text {Meet }}, \mathrm{R}_{\text {Disjoint }} \mathrm{T}_{\text {Coveredby }}, \ldots \ldots \ldots\right\}$. The event of land change may cause the spatial change of land parcel. When land change occurs, we will store these new land parcels into Current Land Parcel Set, and store the previous land parcels into Historic Land Parcel Set. In this process, spatiotemporal topological relationships between related land parcels will be changed.
(1) The spatio-temporal topological relationships between land parcel in Current Land Set

Suppose CPi, CPj are two land parcels in Current Land Parcel Set, thus their life span can be expressed as $\mathrm{Li}=(\mathrm{ti}, \mathrm{c})$ and $\mathrm{Lj}=(\mathrm{tj}, \mathrm{c})$, respectively, where $\mathrm{c}$ means the current time in the world. Therefore the temporal topological relationships between $\mathrm{CPi}$ and $\mathrm{CPj}$ are expressed as $\left\{\mathrm{T}_{\text {Cover }}, \mathrm{T}_{\text {Coveredby }}, \mathrm{T}_{\text {Equal }}\right\}$. From the semantics of cadastral management, the spatial topological relationships between $\mathrm{CPi}$ and $\mathrm{CPj}$ are expressed as $\left\{\mathrm{R}_{\text {Disjoint }}\right.$, $\left.\mathrm{R}_{\text {Meet }}\right\}$. Accordingly, we can conclude that the spatio-temporal topological relationships are expressed as $\left\{\mathrm{R}_{\text {Disjoint }} \mathrm{T}_{\text {Cover }}\right.$, $\mathrm{R}_{\text {Disjoint }} \mathrm{T}_{\text {Coveredby }}, \quad \mathrm{R}_{\text {Disjoint }} \mathrm{T}_{\text {Equal }}, \mathrm{R}_{\text {Meet }} \mathrm{T}_{\text {Cover }}, \quad \mathrm{R}_{\text {Meet }} \mathrm{T}_{\text {Coveredby }}$, $\left.\mathrm{R}_{\text {Meet }} \mathrm{T}_{\text {Equal }}\right\}$

(2) The spatio-temporal topological relationships between land parcel in Historic Land Set

Suppose HPi, HPj are two land parcels in the historic land parcels set, the temporal topological relationships between them are any kind of 8 temporal relationships, and the spatial relationships between them are also any kind of 8 spatial relationships. Therefore, the spatio-temporal topological relationships between land parcels in Historic Land Set are any kind of 64 spatio-temporal relationships.

(3) Spatio-temporal relationships between land parcel in Current Land Set and land parcel in Historic Land Set

Suppose CPi is a land parcel in Current Land Set and HPj is a land parcel in Historic Land Set. Their life spans are expressed as $\mathrm{Li}=(\mathrm{ti}, \mathrm{c})$ and $\mathrm{Lj}=\left(\mathrm{t}_{\mathrm{js}}, \mathrm{t}_{\mathrm{je}}\right)$, respectively. Their temporal relationship between $\mathrm{CPi}$ and $\mathrm{HPj}$ is any kind of 8 temporal relationships, and their spatial relationship is any kind of 8 spatial relationships. Therefore, the spatio-temporal relationships between $\mathrm{CPi}$ and $\mathrm{HPj}$ is any kind of 64 spatiotemporal topological relationships.

Based on the above discussion, we know that there are complex spatio-temporal topological relationships. In cadastral database, we always concentrate on the spatio-temporal relationships between land parcels in Current Land Set, and the spatiotemporal relationships between land parcel in Current Land Set and land parcel in Historic Land Set, e.g. the spatio-temporal relationships of the successive land parcel. Therefore, we can conclude that:

(1) If HPi and HPj are two land parcels in Current Land Set, the spatial topological relationships are in $\left\{R_{\text {Disjoint }}, R_{\text {Meet }}\right\}$, and the spatio-temporal topological relationships are in $\left\{\mathrm{R}_{\text {Disjoint }} \mathrm{T}_{\text {Cover }}\right.$,

$\mathrm{R}_{\text {Disjoint }} \mathrm{T}_{\text {Coveredby }}, \quad \mathrm{R}_{\text {Disjoint }} \mathrm{T}_{\text {Equal }}, \mathrm{R}_{\text {Meet }} \mathrm{T}_{\text {Cover }}, \mathrm{R}_{\text {Meet }} \mathrm{T}_{\text {Coveredby }}$, $\left.\mathrm{R}_{\text {Meet }} \mathrm{T}_{\text {Equal }}\right\}$.

(2) If $\mathrm{Pi}$ and $\mathrm{Pj}$ are two land parcels in cadastral database, and the spatio-temporal relationships of between them are in $\left\{R_{\text {Overlap }} T_{\text {Meet }}, R_{\text {Cover }} \mathrm{T}_{\text {Meet }}, R_{\text {Coveredby }} \mathrm{T}_{\text {Meet }}, \mathrm{R}_{\text {Equal }} \mathrm{T}_{\text {Meet }}, \mathrm{R}_{\text {Inside }} \mathrm{T}_{\text {Meet }}\right.$, $\left.\mathrm{R}_{\text {Contain }} \mathrm{T}_{\text {Meet }}\right\}$, e.g. spatio-overlap and time-meet, they are two successive land parcels. For example, Figure. 1(a) shows that land parcel $\mathrm{A}$ and land parcel $\mathrm{B}$ are $\mathrm{R}_{\text {Cover }} \mathrm{T}_{\text {Meet }}$, they are a pair of successive land parcels.

\section{CONCLUSIONS}

There are complex spatio-temporal relationships among cadastral entities. In the paper, we discuss and develop an integrated representation of spatio-temporal topological relationships within a 3-dimensional temporal space. And we conclude the possible spatio-temporal topological relations between land parcels. The resulting spatio-temporal relationships between land parcels may be used to validate spatio-temporal relationships if they are explicitly stored in the database, or otherwise to compute and discover them. It is beneficial to query and updating of land parcels. Future work 
concerns the computation and discovery of spatio-temporal relationships between land parcels in cadastral database.

\section{ACKNOWLEDGEMENTS}

This research is supported by the National Natural Science Foundation of China (No.41101372), the Scientific Research Starting Foundation for High-level Personnel of North China University of Water Resources and Electric Power, the Natural Science Foundation of the Education Department of Henan Province (No.2011B42003), and the Natural Science Foundation of Zhengzhou City (No.10PTGS507-8).

\section{References from Journals:}

Allen James F, 1984. Towards a General Theory of Action and Time, Artificial Intelligence, 23, pp.123-154.

CAO Zhiyue, LIU Yue, 2002. An object-oriented spatiotemporal data model, Acta Geodaetica et Cartographica Sinica, 31(1), pp.87-92

CHANG Zheng, CHEN Jun, DU Daosheng, 1997. Cadastral spatio-temporal database organization and query based on properties of land subdivision, Journal of Wuhan Technical University of Surveying and Mapping, 22(3), pp.216-221

Claramunt Christophe, Jiang Bin, An integrated representation of spatial and temporal relationships between evolving regions, Geographical systems,(2001)3:411-428.

Egenhofer Max J, Franzosa Robert D, 1991. Point-Set topological spatial relations, International Journal of Geographical Information Systems, 5 (2), pp.161-174.

FAN Yating, YANG Jianyu, ZHU Dehai, 2009. The development of spatio-temporal data model in cadastre management, Science of Surveying and Mapping, 34(5), pp.139141

FENG Hangiian, MA Tuhua, LIU Weihong, LI Wei, 2006. Topology relationship analysis of cadastral spatial database and validity method based on rule, Computer Applications, 26(10), pp. $2522-2523$

WANG Kanghong, ZHONG Ershun, 2001. The analysis on time successions of cadastral entities, Geographical research, 2001, 20(3), pp.372-379.

SHEN Jingwei, WEN Yongning, LV Guonian, et al, 2010. Representation and Reasoning about Spatial-Temporal Topological Relationships, Geography and Geo-Information Science, 26 (6) ,pp.1-5

SHU Hong, CHEN Jun, DU Daosheng, FAN Qibin, 1997.Definition of Spatio-temporal Topological Relationships and Description of Temporal Topological Relationships, Acta Geodaetica et Cartographica Sinica, 26 (4) , pp.4299-306

XU Zhihong, BIAN Fuling, 2002. Cadastral spatial entity and its spatio-temporal topology relationship, Geomatics and Information Science of Wuhan University, 27(5), pp.522-527

WU Changbin, CHEN Xia, 2012.Representation and detection of parcel spatio-temporal topological relationships, Journal of PLA University of Science and Technology (Natural Science Edition), 13(1), pp.51-56
XUE Cunjin, SU Fenzhen, 2008. Research on Spatio-temporal Topologies Based on Cartesian Operations, Computer Engineering and Applications, 44(21), pp.20-24

ZENG Lianbin, SONG Yingchun, 2010. Calculation and search for the spatio-temporal topological relation of spatio-temporal object, Journal of Hunan University(Natural Sciences), 37(4), pp.85-89

ZHOU Xiaoguang, CHEN Jun, JIANG Jie, ZHU Jianjun, 2003. Topological relations between parcels, Acta Geodaetica et Cartographica Sinica, 32(4), pp.356-361 